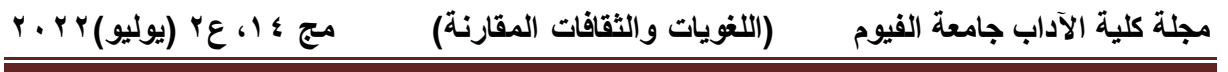

\title{
Cécile Portier : jeux de poétique en ligne
}

\author{
Dalia Metawe* \\ dalia.metawe@yahoo.com
}

\section{Résumé}

Cécile Portier est l'un des auteurs français qui écrivait sur Internet il y a plusieurs années et diffusait ses projets numériques sur son blog petiteracine.net, un site internet qu'elle a créé pour écrire et publier ses œuvres numériques. Elle écrit également dans des revues de littérature en ligne telles que Contretemps, D’ici là, Remue.net et des sites web de fiction. Cette recherche vise à étudier comment et dans quelle mesure le numérique a modifié la pratique d'écriture de Cécile Portier, ses habitudes et gestes quotidiens, et comment il affecte la structure et la texture de ses créations. À cette fin, nous aborderons les créations numériques de $\mathrm{C}$. Portier tout en examinant la façon dont elle explore le monde réel rempli de signes et de traces, et gère l'espace numérique dans ses créations. Nous étudierons également les nouvelles formes d'écritures et les modalités de littérarité, ainsi que les jeux de poétique que le numérique lui permet de mettre en œuvre sur Internet.

Mots-clés : Cécile Portier, numérique, écriture, stylistique, poétique.

\footnotetext{
*Professeure adjointe - Faculté des lettres - Université de Menoufia.
} 


\section{Introduction}

L'environnement numérique dans lequel nous vivons depuis plus de trente ans incite de plus en plus les auteurs à créer des œuvres littéraires en ligne en considérant Internet comme un atelier de production, de discussion, de lecture et d'édition. Cécile Portier est l'un des auteurs français qui écrivait sur Internet il y a quelques années et diffusait ses projets numériques sur son blog petiteracine.net, un site internet qu'elle a créé pour écrire et publier ses projets numériques, dans des revues de littérature en ligne telle que Contretemps, D'ici là, Remue.net et dans des sites web de fiction. Passionnée par les méthodes numériques, elle a souligné qu'à travers l'écriture numérique, son travail a développé en exploitant pleinement les potentiels du web qui l'ont amenée à d'autres formes d'art auxquelles elle n'avait jamais pensé (Cécile Portier, s. d. "A").

Cette recherche vise à étudier comment et dans quelle mesure le numérique a modifié la pratique d'écriture de Cécile Portier, ses habitudes et gestes quotidiens, et comment il affecte la structure et la texture de ses créations. À cette fin, nous aborderons les créations numériques de C. Portier tout en examinant la façon dont elle explore le monde réel rempli de signes et de traces, et gère l'espace numérique dans ses créations. Nous étudierons également les nouvelles formes d'écritures, les modalités de littérarité, ainsi que les jeux de poétique que le numérique lui permet de mettre en œuvre sur Internet. Notons que l'activité d'écriture de Cécile Portier travaille les formes papier, numérique et performance. Dans cette étude, nous examinerons seulement ses écrits nativement numériques ${ }^{\mathrm{i}}$.

Alors que la numérisation envahit rapidement notre vie, nos activités et nos habitudes quotidiennes, et le passage prévu de la culture du livre à la culture de l'écran, de nombreux termes 
liés à l'informatique ont été ajoutés à notre glossaire. Parmi eux, Guénaël Boutouillet a conçu le terme « auteur numérique »en 2013. À ce propos, certaines questions se posent: Qu'est-ce qu'un auteur numérique? Existe-t-il vraiment? Appelons-nous chaque auteur numérisé $e^{i i}$ un auteur numérique? En pratiquant l'écriture sur le web, Cécile portier est-elle un écrivain numérique?

\section{Cécile Portier : auteure numérique}

Il existe de plus en plus d'écrivains ${ }^{\mathrm{iii}}$ qui pratiquent largement l'écriture sur Internet et réalisent leurs œuvres sur leurs blogs. Guénaël Boutouillet, par souci de catégorisation, s'est interrogé sur l'existence d'écrivains dits «numériques» (2013). Il affirme que cette question: «se pose, redoublée, à l'écrivain, affecté, comme tout individu, dans ses faits et gestes quotidiens, mais aussi au cour de son activité : l'atelier de production, les canaux de diffusion, comme les lieux de destination, d'usage, de ses textes ont déjà changé et continuent de muter » (Ibid). Alors, un tel écrivain ainsi numérisé deviendra-t-il un écrivain numérique ? Boutouillet explique que non, et dans la plupart des cas, ce changement de paradigme global ne semble pas affecter tous les travaux en cours, comme si «externe» ne joue pas de rôle en interne, ou joue rarement, et comme s'il semblait possible d'isoler l'écriture du monde informatique actuel. Cependant, dans cet environnement numérique, un grand nombre de poètes et de romanciers, saisissant les fluctuations de l'extérieur, reconsidèrent l'apparente réalité que le numérique affecte la structure et la texture des textes.

Dans ce contexte, la manière dont nos vies et leurs représentations altérées par la «mise en données» attirent, interrogent et agissent C. Portier qui a investi les possibilités offertes par la technologie numérique dans ses travaux. Elle a 
affirmé que le numérique va, pour elle, dans le sens où c'est le seul «bureau», la seule «salle d'écriture » qui puisse établir et réaliser son désir d'écriture puisqu' elle n'a pas beaucoup de temps et d'espace, et elle écrit dans leur interstice sur son téléphone, dans des fichiers dropbox qui peuvent être récupérés de n'importe où (Portier, s. d. "A"). Ses lieux de production, diffusion et de lecture deviennent ainsi numériques. Le web lui a donné l'occasion d'écrire «dans un espace public » où il n'y a pas de distance entre les lecteurs/internautes qui entrent librement, se réunissent, se reconnaissent en discutant tout le temps sans être obligé d'avoir des autorisations pour y entrer. Ils peuvent également discuter avec les écrivains et commenter leurs travaux. Portier admet que l'écriture en ligne a changé sa pratique stylistique, car le numérique, pour elle, n'est pas seulement un médium ou un outil, mais il est indéniablement un nouveau domaine qui mérite d'être investi et questionné. (Ibid.). Selon cette perspective, Portier, «usant de moyens numériques pour interroger le numérique et ses moyens, semble pouvoir être considérée, en plusieurs endroits, comme une auteure numérique » (Guénaël Boutouillet, 2013).

Portier a publié plusieurs projets en ligne sur son blog Petite Racine (www.petiteracine.net) comme La tête que ça nous fait, Dans le viseur, À mains nues, Compléments d'objets, Ni l'un ni l'autre, Traque traces, Étant donnée. Quand on navigue sur son blog, l'internaute trouvera la page d'accueil dans laquelle les facettes supérieures le dirigent vers ses différentes œuvres numériques, une image des clous implantés dans le mur est présentée ci-dessous, comme le montre la figure (1). 


\begin{tabular}{|c|c|c|}
\hline 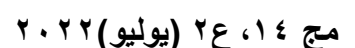 & (اللغويات و الثقافات المقارنة) & مجلة كلية الآداب جامعة الفيوم \\
\hline
\end{tabular}

\section{Petite Racine}

Ici c'est fait pour écrire

la tête que ça nous fait dans le viseur compléments d'objets a mains nues singeries ni l'un ni l'autre traque traces étant donnée
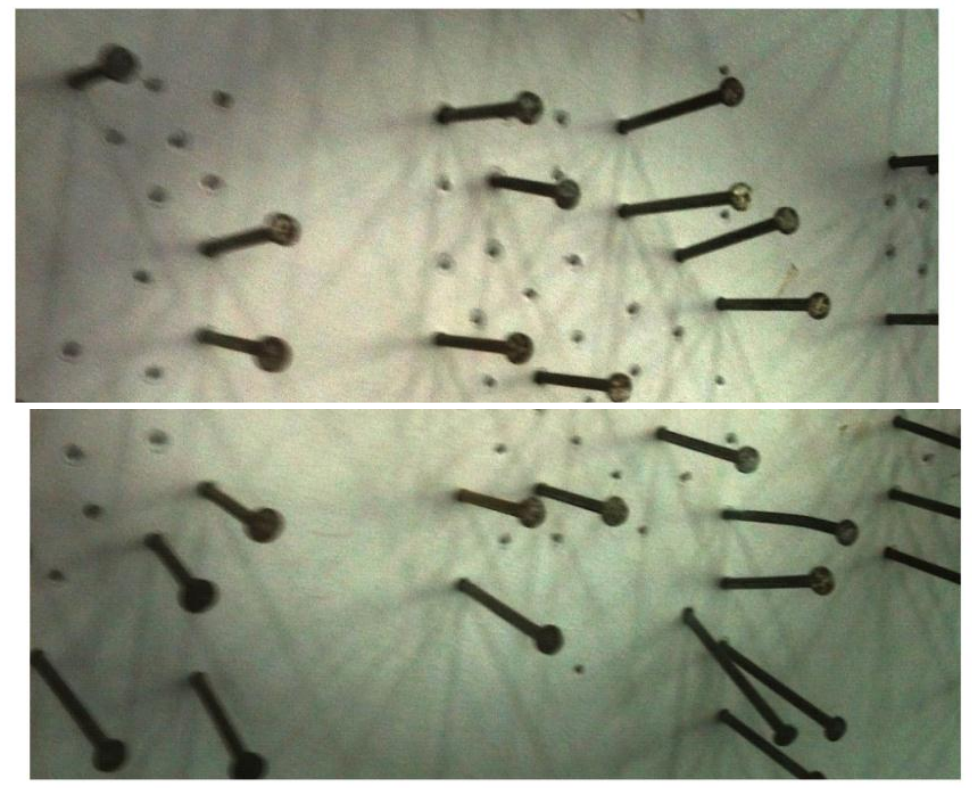

Artides récents

- Pourquoil le masquer

- Devenir dindon

- Reverondre, et remercier

Site d'ecriture

Fig. $(1)^{\text {iv }}$

Portier explique pourquoi elle a choisi ces termes Petite Racine pour être le nom de son blog :

«- Pour la fleur des pendus

- Pour celle, mathématique, qui ne tourne pas rond

- Pour celle qu'on enfouit, afin qu'elle refleurisse plus tard, peut-être, et multipliée

- Pour celle, nerveuse, faisant que par le même chemin passe douleur et délice » (www. Petitracine.net).

Cette auteure dédie à travers un passage poétique son blog aux fleurs des pendus, mystérieuses et magiques fleurs de mandragore que les médecins chinois anciens et antiques ont connues pour leurs vertus anesthésiques et narcotiques ${ }^{\mathrm{v}}$. La racine de ces fleurs

(Cécile Portier : jeux de poétique en ligne...) Dr. Dalia Metawe 
ressemble vaguement à un corps humain. C. Portier cite également le poème d'Henri Michaux «Portrait des Meidosemmes » paru dans son recueil La vie dans les plis (1948) (Cf. www. Petitracine.net.). Ce portrait, conçu comme le portrait du poète lui-même, donne accès à l'espace du dedans de Michaux (R. Deslauriers, 2002). Par conséquent, en termes d'exploration de Portier de notre espace privé interne, la citation du poème de Michaux est d'une grande importance. Dès ses premiers travaux, elle s'intéresse à l'écriture de «nos vie à notre insu » (Portier, s. d. "C"). Cette auteure concentre particulièrement sa vision et ses œuvres sur le corps humain (tête, visage, mains, signes) et les lieux cachés car très intimes. C'est un voyage pour comprendre notre connaissance de soi, nos traces et l'environnement qui nous entour. Nous abordons à présent les cuvres numériques de Portier tout en mettant en relief les jeux de poétique que les potentialités numériques lui permettent de créer en ligne.

\section{Exploration des visages intérieurs : poétique de l'intime}

La tête que ça nous fait (2014-2015) présente la première facette sur le site Petite Racine de C. Portier. Cette création numérique vise à explorer nos visages intérieurs qui reflètent nos sentiments les plus intimes. Sur la page d'accueil de l'œuvre, les lecteurs trouvent plusieurs dessins caricatures de visage, en dessous desquels sont écris des mots (verbes) correspondant aux images, par exemple, visage lumière correspond au éblouir, ou bien visage de chouette harfang pour résister, comme le montre la figure (2). 

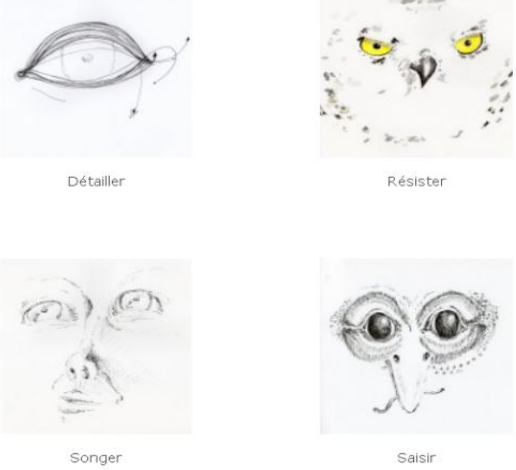

Fig. $(2)^{\mathrm{vi}}$

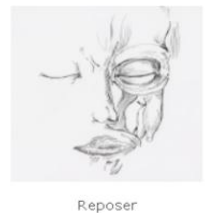

Reposer

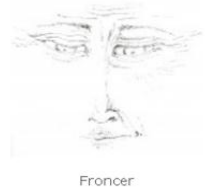

Froncer
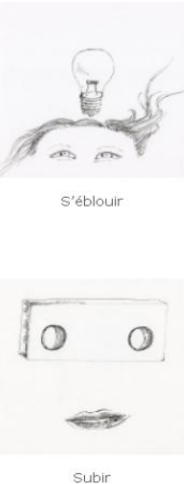

Le lecteur/internaute aborde d'abord cette œuvre numérique visuellement à travers les dessins caricatures qui constituent la première entrée de l'œuvre. Cette partie visuelle joue un rôle marqué pour la perception de l'œuvre car les expressions faciales (en particulier la forme des yeux) soulignent la signification des verbes écrits ci-dessous, ce qui rend cet objet numérique unique. En cliquant sur le verbe sous l'image, un texte (petit texte pour la plupart des dessins) s'affiche dans lequel Portier décrit les différents émotions et traits qui traversent nos pensées et apparaissent sur nos visages. Le style poétique de Portier et les descriptions détaillées et méticuleuses permettent de scruter attentivement tous les détails émotionnels et les sentiments intimes de l'auteure découlant de son expérience personnelle, comme le montre l'entrée suivante intitulée « songer » (2014): 


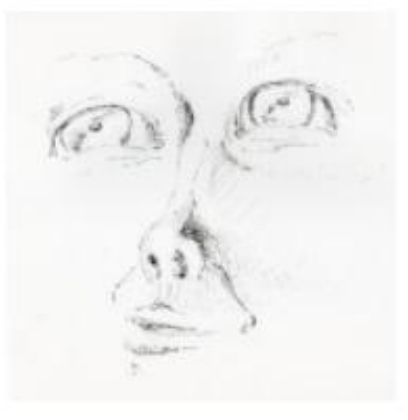

Songer

"Parfois quelque chose nous renverse, sans heurt, et même sans vertige. Disons sans violence. Et même sans fatigue. C'est juste que le derrière de la tête devient beaucoup plus lourd et doit s'abandonner dans le profond des coussins, comme on s'ancre. Ainsi horizontalisés, les yeux ne voient plus, reflètent seulement, nuages ou néons du plafond, c'est selon, c'est égal.

On a exactement la même tête que d'habitude, sauf qu'on n'y est plus. On flotte un peu partout autour. Et encore, flotter, c'est beaucoup trop de stabilité encore, pour dire comme les pensées sont fluides, liquides, dégelées enfin de leur forme - débitée en cubes - des jours où la tête est droite et le cou raide.

Car oui, ça chahute, il y a du remous, la ligne de flottaison n'est pas stable. De temps à autre le néon radieux se voile d'un souci traversant. L'instant d'après c'est beau fixe depuis l'éternité, et c'est bien parce qu'on sait enfin dans ces moments là, que rien ne dure ».

La poéticité de ce texte réside dans la finesse de l'expression, la précision de la description, la clarté de vision et le langage parsemé d'images et de métaphores, mettant en relief l'intimité de l'acte de réfléchir. Remarquons que Portier indique certains mots clés (tags) en dessous de chaque fragment textuel pour aider le lecteur à percevoir le sens voulu. Le lecteur saute d'un dessin à l'autre en simple clic, grâce aux deux mots anglais avec deux flèches situées aux côtés de l'écran : earlier et more. Cette organisation cyclique fait que le lecteur ne s'arrête jamais à 
explorer le contenu de cette création numérique qui mêle constamment texte et dessins mettant en œuvre une poétique de l'intime extrêmement particulière. Par ailleurs, l'interaction de dessins et de commentaires textuels renforce la perception de l'émotion décrite.

\section{Poétique des traces}

Dans la mesure où «nos vies s'écrivent par traces, que nous laissons sans y penser, mais qui nous documentent et nous archivent » (Portier, 2015), Portier a abordé le thème des traces dans plusieurs de ses créations numériques, dont Dans le viseur, Traque trace, Étant donnée. La trace, selon Portier, est ce genre d'inscription, qui établit une relation causale entre l'auteur et sa trace d'une manière particulière. Pour elle, remonter à la source est toujours un travail incertain. Cependant, dans la méthode de suivi numérique, ce type d'incertitude n'existe plus, ou rarement, ce type de suivi peut être retracé jusqu'à l'adresse sans erreur. La trace est «ce qui vibre en permanence-comme une corde tendue entre deux pôles. Et c'est cette vibration (tension, ambivalence), qui est aujourd'hui circonvenue dans l'organisation de nos traces en données numériques » (Portier, 2016). D'un point de vue définitionnel, cette auteure a expliqué que les traces peuvent être obscurcies, qu'elles deviennent un élément clé de la traçabilité, il n'y a aucun doute sur cette manière d'écrire. Dans cette nouvelle définition des traces, ce que nous nions, selon elle, c'est la possibilité d'autres causes. (Portier, 2015).

Dans le viseur (2012-2015), l'auteure, à partir des photographies éparses (figure 3), explique pourquoi les traces numériques qu'on laisse chaque jour sont différentes : «La trace, dans l'acception commune (passée?), est cette tension entre ce qui reste d'un temps qui n'est plus, ce qui insiste de ce temps 


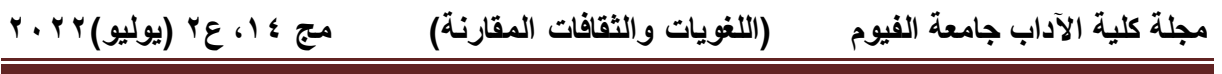

révolu, et ce qui, de ce qui est passé et inscrit, est voué à s'effacer. La trace est une présence, qui est le témoignage de quelque chose qui n'est plus, d'une absence. A cela, à cette indécidable présencelabsence, se substitue, dans le régime de la trace numérique, la notion d'une disponibilité infinie des inscriptions qui nous concernent et nous décrivent »(Ibid.).

DANS LE VISEUR

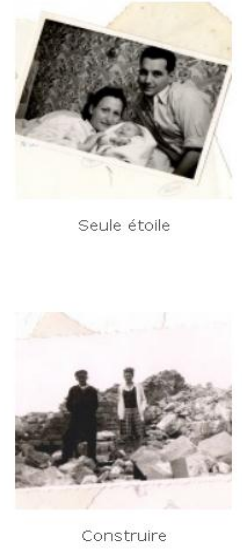

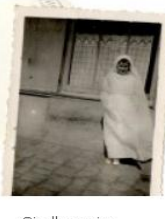

Si elle arrive

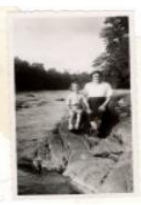

L'otage

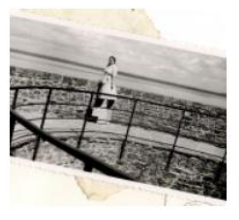

Juchée

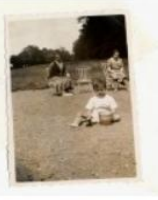

L'attente

\section{Fig. (3) vii}

Portier a indiqué dans la rubrique «Jauchée » (2012) qu'elle a trouvé ces photos dans un bac au marché seconde main de la photo de famille. À partir de ces photos perdues, l'auteure a retracé l'histoire de leurs propriétés. Elle est sortie du document et rentrée par l'œil, par le je, dans la fiction. Le lecteur/internaute voit d'abord une série de photos muettes et immobiles en noir et blanc. Comme le précise l'auteure, les personnages dans les photos sont morts à l'heure où le lecteur les regarde. Personne ne les connait. Elle n'a que leurs photos. La seule trace qui reste de ces personnes inexistantes ce cercle des souvenirs disparus.

Portier s'est mise dans la peau du photographe qu'il a pris ces photos et a imaginé les histoires et les traits des personnages sur les photos. Ces gens prennent vie sous les yeux du lecteur, 
s'animent, ont des sentiments, des histoires, des proches grâce aux textes fictifs de Portier situés au dessous des photos. Dans cette œuvre numérique, la fiction joue un rôle remarquable car elle assure la seule continuité possible de ces photos et les rend présentables, surtout avec les indices extrêmement faibles et peu de contexte montrés dans ces photos. Après avoir lu les textes, et en regardant à nouveau ces photos, impossible de les voir de la même façon, avec le même œil. Portier a expliqué la raison pour laquelle elle a choisi la fiction : "Pour moi la fiction ce n'est pas forcément une histoire, un récit, dans lequel vouloir insérer à toute force la photographie, comme pour lui redonner une place, à partir de laquelle elle soit crédible, elle soit lisible. Une vie, une vie vécue, on ne la vit pas comme un roman. Une vie vécue est rarement crédible, jamais lisible. Bien sûr on peut toujours remplir. Mettre des mots sur ces bribes de vie, pour les rendre présentables, comme on appose de la cire molle au visage des morts accidentés.[...] La seule continuité possible pour ces photographies sans propriétaire, je voyais bien que c'était la fiction, mais pas forcément le récit. Quelque chose plutôt, de lordre du dialogue, de la poésie. Quelque chose où l'on est plus impliqué » (Cécile Portier, s. d. "B").

En leur donnant une vie, le lecteur partage avec Portier son ressenti par rapport à ces fantômes de manière très sensible. Ainsi la réalité de l'existence de ces photos se mêle à l'imagination et à la fiction. L'originalité de cette création numérique réside dans le fait que les personnages sur les photos subsistent à travers des mots, pas forcement du récit, mais d'enchaînement de dialogues, de poèmes en prose poétique et de poésie. Prenons par exemple la photo ci-dessous : 


\begin{tabular}{|c|c|c|}
\hline 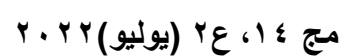 & (اللغويات والثقافات المقارنة) & مجلة كلية الآداب جامعة الفيوم \\
\hline
\end{tabular}

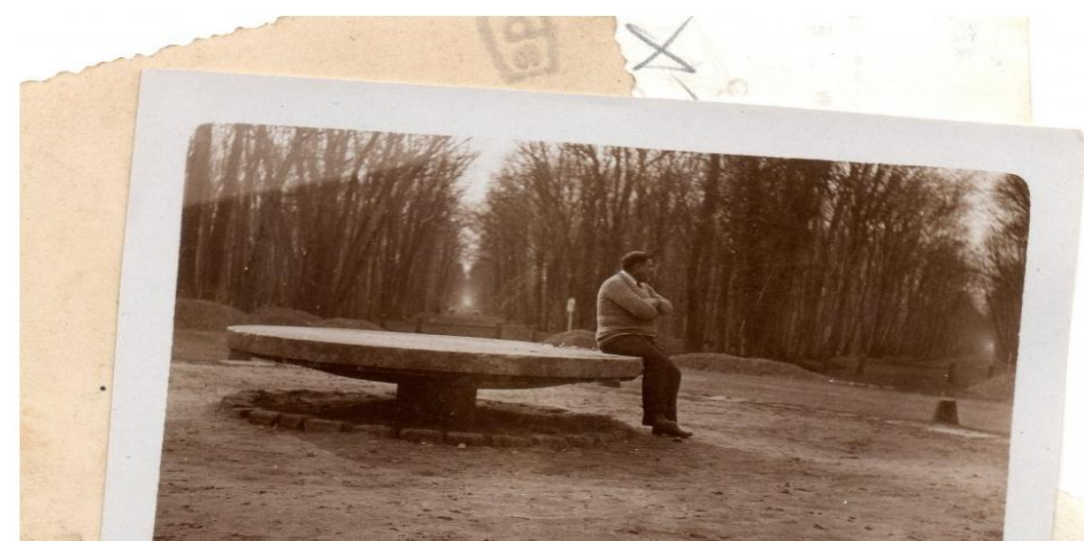

Fig. (4) viii

«Ta vie les bras croisés, à attendre et regarder. Tu es fatigué maintenant, et pas tellement d'avoir marché. Tu t'engonces en toi-même, considérant ces trouées de lumière entre les arbres comme autant de futurs qui ne sont plus pour toi. La table d'orientation, tu ne la regardes pas, tu t'assieds dessus. Qu'importe, sembles-tu penser. Toutes les allées se ressemblent. Tous les chemins reviennent, et ne mènent pas si loin. Es-tu essoufflé? As-tu mal aux pieds? Sans doute même pas. La terre est battue, foulée, moelleuse elle aussi, d'être vaincue. Tu as les bras croisés, jouissant tranquillement d'être là, à considérer la borne qui te fait face. Ta vie de profil, à ne jamais regarder l'horizon en face. Tu as pris place, depuis longtemps semble t-il, sur un tourniquet vertigineux, d'être à ce point immobile».

Ce poème en prose ne raconte pas d'histoire ni transmet d'informations, mais c'est un dialogue cherchant un effet poétique. En lisant ce langage poétique émouvant, les lecteurs seront attirés par le style de l'auteure qui relie les morts aux vivants. Le pouvoir du style de Portier est de faire croire aux lecteurs que c'est la véritable histoire du personnage, même s'il sait que c'est l'imagination de l'auteure. Ce texte change la façon dont les lecteurs regardaient initialement ces photos. Cela les fait vibrer, bouger, être émus, rire et réfléchir. En découvrant, 
les unes après les autres, de photos, de nouveaux personnages, de nouveaux décors, de nouvelles vies, cette création numérique montre comment la vie s'est diversifiée, différente et pourtant entrelacée. Comme le souligne Guénaël Boutouillet, Portier a affronté dans cette série de photos le paradoxe corporel devenu trace. Un ensemble de vestiges, incarnés comme des données. Puis, elle s'est interrogée sur cette vie abstraite avec un nouveau renversement de la logique ordinaire (2015).

Ainsi, Dans le viseur tisse une relation immanente entre image et texte dans la mesure où le texte donne vie à l'image et non l'inverse. Portier a travaillé sur deux registres qui ne semblent pas liés : les données, pouvant être collectées par quelqu'un sur le serveur et sur Internet, et les photos d'une personne, stockées dans son tiroir, son armoire, son disque dur, et pour certaines d'entre elles peuvent être trouvées dans l'état des données, stockées numériquement et mises sur Internet. Par conséquent, cette œuvre numérique réinvente une vie à partir de ces minuscules traces, en l'absence de tous renseignements disponibles. Elle veut questionner cette écriture de l'extérieur de nous, non pas à partir du moment où ces photos ont été prises, mais à partir du moment où elles doivent être considérées comme des traces et des vestiges (Portier, s. d. "C") : «Nous vivons une période où les fragments épars de ce qui est sensé nous décrire sont si nombreux, si disparates, que pour les faire parler ensemble, et retrouver dans cette polyphonie quelque chose de la personne captée, nous devons faire une sorte d'archéologie en temps réel » (Ibid.).

Quant à Étant donnée (fig. 5), cette création numérique interactive inspiré du célèbre ouvrage de Marcel Duchamp Étant donné, est un récit transmédia sous forme de site $w^{2} b^{i x}$. Le début de l'histoire est simple : une femme nue a été retrouvée dans un endroit vacant, elle souffrait d'amnésie et illuminait la journée 


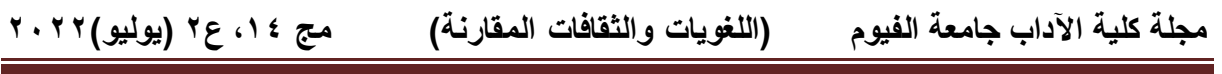

avec une lampe à gaz à la main. Le site Web invite les lecteurs/internautes à regarder cette femme à travers un trou dans le grillage comme un voyeur, et à utiliser les données numériques laissées par la femme allongée dans sa vie antérieure pour la rhabiller.
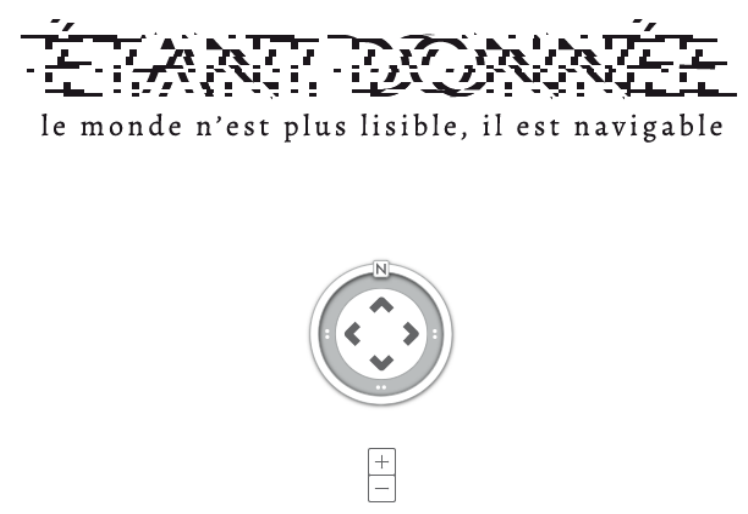

Fig. $(5)^{\mathrm{x}}$

Sur la page d'accueil de cette œuvre, la boussole interactive joue un double rôle : elle aide les lecteurs/internautes à naviguer dans l'œuvre tout comme il circule dans un espace réel, en revanche, l'image dépasse cette simple fonction apparente, avec le soustitre «Le monde n'est plus lisible, il est navigable » en jouant un rôle visuel en tant que sous-texte numérique et spatial. Par conséquent, cette boussole constitue le seuil de la réalité et l'icône de la visualisation de l'œuvre. Ainsi, la naturalisation des signes et symboles visuels permet aux lecteurs/internautes de se plonger immédiatement dans un espace réel au lieu d'une simple histoire.

Portier a déclaré que les données d'aujourd'hui promettent qu'on pourra tout voir, et il est facile d'oublier qu'il y a un «point aveugle ». Dans cette vision gagnante, les choses ne sont pas si claires. Cette situation nécessite un autre type de lumière, 
poétique, politique, afin de rompre avec l'idée évidente du «tout est donné » (Portier, 2016). Cette œuvre numérique est une enquête poétique sur nos traces numériques, et elle prend la forme d'un projet d'art mixte. Elle comprend une série de textes et de créations diverses, dont des «poèmes vidéo », qui vise à reconstituer l'histoire de survie de cette femme amnésique. Cependant, ce récit n'est pas conforme à la crédibilité et à l'intégralité de nos récits officiels dans notre vie axée sur les données : il tente de laisser des blancs, de transférer des traces et de poétiser cette fiabilité pour la déjouer. Comme le souligne Gaëlle Debeaux qu'Étant donnée est un projet de «ressaisissement» qui reprend nos traces via les outils numériques. Il vise à réintroduire par l'écriture et la fiction les traces comme condition de notre traçabilité, et l'incertitude des traces comme condition de notre liberté (2019).

Par ailleurs, l'image numérique dans cette œuvre est imbriquée ainsi que sa performativité spatiale pour produire de l'espace employant une des applications numériques connues, Google Maps. Portier nous présente une nouvelle vision de la formation spatiale à travers l'utilisation littéraire des potentialités des images numériques, tenant compte aux profonds changements survenus avec l'avènement des nouvelles technologies numériques surtout le web qui apporte généralement des modifications spatiales mettant en lumière une perception renouvelée de l'espace (E. A. Marchese, 2020 : 5-8; Schmitt, $1985: 52$ ).

Dans la fiction urbaine Traque Traces ${ }^{\mathrm{xi}}$ (fig. 6), l'idée d'écrire à partir des données qui nous écrivent réapparaît. Elle consiste à reconstruire une cité imaginaire sous la vraie cité et à faire vivre et prospérer toute la population : "Nous avons donc écrit un peuple. Un tout petit peuple, qui habite où nous habitons: Aubervilliers, Nord Paris. Chaque personne 


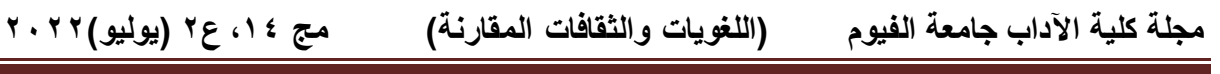

constituant ce peuple a laissé en vivant des traces que cette fiction trace et retrace pour vous » (Guénaël Boutouillet, 2011). Le but de ce roman est donc d'utiliser les données pour jouer à des jeux d'arrosage, écrire nos données, refaire leur rôle d'analyse sérieuse et apprendre à lire cette nouvelle écriture par les données

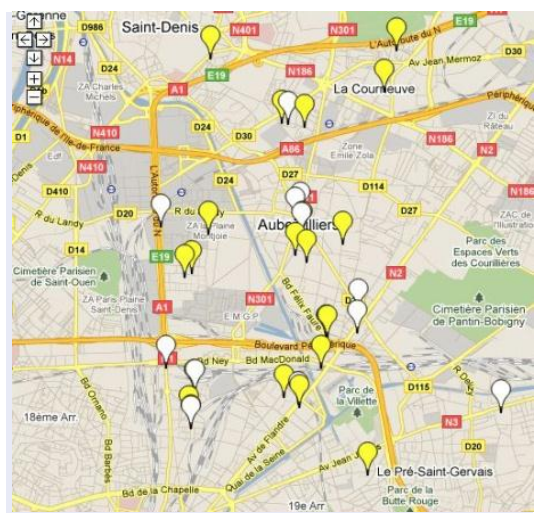

Fig. (6) $)^{\mathrm{xii}}$

Comme le montre la figure (6), cette œuvre est un site qui emploie le même procédé poétique qui convertit la cartographie numérique en technique littéraire et poétique. L'auteure intègre la fonction de Google Maps dans la structure littéraire des personnages et la relation entre eux, afin de produire une œuvre numérique qui regroupe l'espace physique des personnages, l'espace de navigation Web et l'espace de discours narratif.

Portier exploite ainsi les possibilités numériques pour créer une écriture des traces et construire des espaces à travers des concepts innovants de construction spatiale, en donnant à ses œuvres numériques une dimension sinon physique, sûrement spatiale. L'auteure a utilisé des procédés poétiques et littéraires, tout en rendant iconiques les codes de la cartographie numérique contemporaine, comme le point rouge «vous êtes ici »dans Traque Traces ou l'interface typique de Google dans la section disparition dans Étant donnée. Dans cette perspective, Fanny 
Siaugues a affirmé : "L'écriture numérique crée un nouveau domaine spatial qui ré-organise notre positionnement corporel, notre attitude réceptive, en tant que corps devant un écran, devant une audio-vidéo, devant un texte, devant un livre. Rappelons les paroles de Bon à nouveau : Plus que jamais, même avec le numérique, le corps écrit » (2019).

\section{Poétique du réel}

Portier invite les lecteurs/internautes à observer le monde à travers les objets dans Compléments d'objets (2009-2014) qui est une autre facette de son blog Petite Racine. Comme son nom l'indique, cette œuvre numérique part des objets du quotidien qui nous entourent, analysant ce qu'ils expriment et représentent et leur impact sur la façon dont nous agissons dans le monde. En liant l'espace numérique à son milieu de vie, l'auteure a une perspective différente, à la fois pleine de curiosité, de critique et d'insolite: "Jouer du fait que sous le soleil les choses n'ont, selon l'heure, pas les mêmes couleurs. Jouer du mystère de la pleine lumière, expliciter dans le sombre. Et puis découper, recomposer. Petits rayons dardés, trompés, qui font que le monde usé, par nous-mêmes salivé, nous redevienne méconnaissable (rubrique «Regarder beau», 2010). Dans une autre rubrique intitulée «Enfiler les perles», elle explique que les objets sont «pris pour leur mystère d'objet, qui ne tient pas à leur beauté mais à l'indéchiffrable écart entre ce pour quoi ils ont été fabriqués et ce qu'au final on attend d'eux » (2010). Ce monde d'objets constitue une des vies que Portier invite à observer d'une autre manière dans la mesure de ce qui enfreint la norme standard et apparait invisible lorsqu'il est utilisé mécaniquement.

Dans la même lignée de Dans le viseur, portier mêle texte et photographie (fig. 7). Elle voit ainsi le monde à partir d'objets en l'éditant à partir d'images et de textes poétiques. Dans cette 
œuvre, la photo d'un objet ou d'un lieu figure sur la page web, suivie d'un fragment textuel occupant la position de la légende de la photo, mais cela va bien au-delà de ce rôle. Au bas de la page se trouve le titre de chaque section, avec une série de tags (mots-clés), qui résument brièvement la photo et le texte.

COMPLÉMENTS D'OBJETS

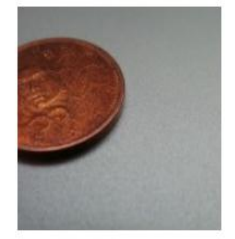

Gagne ma langue

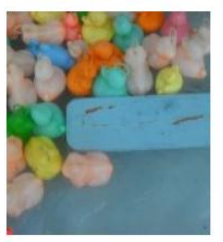

Petit crochet

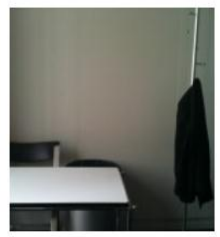

L'hôte

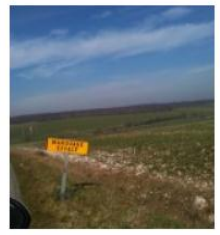

En pointillés
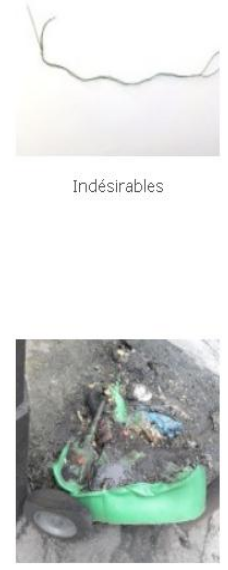

Tout est en place

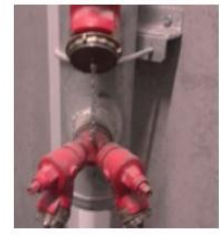

Serveuse vocale

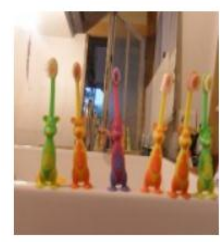

Les inaperçues

Fig. (7) $)^{\text {xii }}$

Dans ce projet, l'auteure met en évidence la relation entre le monde, l'espace et la réalité matérielle, en traitant ces aspects du point de vue de l'agencement des objets et de la possibilité d'appréhender la réalité à travers l'écriture, et insiste sur l'écriture $\mathrm{du}$ «je » écrivant et de son environnement. Comme le remarque Béatrice Lefebvre-Côté: «Portier cherche à rompre l'anonymat des images et à s'approprier le monde qui l'entoure, dans un mouvement allant $d u$ domaine privé à l'espace numérique, questionnant le monde, le réel et l'écriture. Mis en relation avec la théorie de Carl Schmitt sur le nomos et celle de Henri Lefebvre sur la production de l'espace, le travail de Cécile Portier apparaît une instance productrice d'espace, qui permet le décloisonnement de façons littéraires, matérielles et utilitaires 
d'agir sur réel» (2018). Par conséquent, cette création numérique n'est pas seulement une entrée dans l'intimité de l'auteure, mais elle démontre un espace privé, incluant la vie de la famille, de la ville et de la classe ouvrière, que l'auteure questionne afin de mettre en relief des pratiques sociales et matérielles. Du fait de ce questionnement sur la vie quotidienne, les œuvres littéraires de Cécile Portier deviennent non seulement une manière d'écrire la réalité, mais créent également une réalité aussi tangible que les objets qu'elle a photographiés. Cet enchevêtrement de la vie matérielle et de la vie numérique permettra de transcender les frontières entre réalité, imaginaire et numérique.

Par ailleurs, Portier présente le monde des objets des plus triviaux aux plus intéressants à travers une vision poétique, démontrant la «beauté des séries et des variations », et décrivant la matière employée de «sophistiquée, brillante, hautaine, noble» (rubrique «Retour à la poussière », 2010). Notons que dans ses travaux, cette auteure s'intéresse aux détails en leur donnant beaucoup d'intérêt : «Les objets sont des détails bourrés de détails, ce qui est un bon point pour eux. J'ai voulu traiter les objets dans leur matérialité, leur sensualité, bien qu'elle soit toute imaginaire puisqu'aucun de ceux décrits de près ici n'a jamais été devant moi. J'ai voulu aussi les traiter comme des symptômes. Chaque objet décrit, dans sa singularité, dit pour moi quelque chose de l'état de notre monde» (Portier, 2018).

Dans ce projet numérique, l'écriture du réel de l'auteure crée une nouvelle façon de comprendre le monde en donnant une existence et une valeur individuelle aux objets, et en invitant les lecteurs à regarder autrement les choses qui les entourent et à les intégrer dans leur quotidienneté non plus en fonction de leurs habitudes, mais en questionnant l'objet et sa fonction. Dans cette œuvre, la préemption des objets photographiés puis commentés 


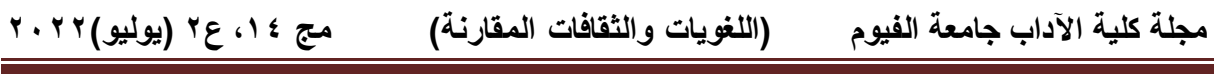

est finalement remplacée par une vision qui perçoit à la fois les choses et leur rapport au monde. Prenons cet exemple de l'entrée intitulée «Les inaperçus » (2011):

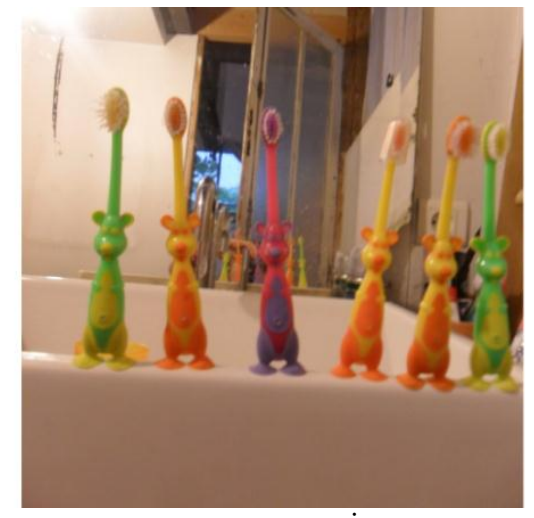

Fig. $(8)^{\mathrm{xiv}}$

«[...] Bref, j'apprends en une image qu'il se passe chez moi, en ma présence, des choses qui m'échappent totalement. Tout un monde parallèle, où les brosses à dents usagées sont sauvées à mon insu du naufrage, et retrouvent en clandestinité celles en service, pour des réunions au sommet. Une sorte de G6, avec photographie sur le perron mais sans mains serrées à la fin. J'ignore si elles sont parvenues à une résolution quelconque, mais je me prends à l'espérer ».

Dans cet exemple, Portier délimite un espace du quotidien à partir de brosses à dent photographiées par sa fille. Notons que le texte est écrit après la capture de la photo et se situe sous l'image. Sa mise en page peut donner l'impression d'un simple texte explicatif. Cependant, à la lecture du texte, le lecteur observera la juxtaposition du langage poétique et de la pensée littéraire avec des objets de la vie quotidienne, mettant ainsi en évidence une pratique spatiale et des espaces de représentation auxquelles la littérature ajoute éloquence et beauté.

Portier présente sa compréhension de la réalité d'une manière singulière, ainsi que sa compréhension unique de l'espace privé et de l'environnement réel. L'écriture du réel de 
cette auteure a donc une fonction poétique pour les objets et les outils quotidiens, mettant en lumière une réflexion sur la littérature et l'existence : elle met d'abord l'accent sur l'objet ou l'espace choisi, puis transforme le texte en matériau poétique et littéraire, s'écartant ainsi de sa fonction normale, comme le montre l'exemple ci-dessus. Elle donne à la réalité des objets une dimension fictive et poétique originalement marquée. Portier affirme que «Pour moi l'écriture c'est toujours mâcher des fragments de réel - parce qu'ils sont difficilement digérables, puis les régurgiter, constater que ces déchets sont beaux, et tenter de les agencer. Il y a donc deux étapes. Une écriture par fragments, par intensité. Puis, de la couture, de la manigance, de la narration $\gg$ (Portier, 5-10-2018)

\section{Contemplations Poétiques}

Une partie de la création numérique de Portier est consacrée à ses observations de la métropole comme dans $A$ mains nues (2009-2011). Cette œuvre numérique rassemble plusieurs photos prises par l'auteure des mains des passagers du métro. L'auteure raconte ses rencontres inattendues avec eux en leur permettant de parler de leurs mains. Àla question posée par un des passagers dans le métro : «[...] alors comme ça vous vous intéressez aux mains», l'auteure explique son objectif en répondant qu'elle s'intéresse aux gens. Elle précise : «Que ce qui me touchait, c'était de voir toutes ces personnes se croiser, s'entasser à parfois se toucher, partager ce même espace, et ne pas même se regarder, se reconnaître, se parler. Que ces espaces dédiés aux déplacements, nous les vivions paradoxalement comme des espaces mornes où rien ne circulait. Que ces transport $n$ 'avaient rien de commun, et que personnellement cela me mettait en colère » (rubrique «Déplacements », 2011). Par conséquent, dans cette œuvre, Portier a envisagé de lire le monde à travers les

(Cécile Portier : jeux de poétique en ligne...) Dr. Dalia Metawe 
mains des gens après avoir lu le monde à travers la tête ou les yeux dans certaines de ses créations numériques, comme nous l'avons déjà montré, considérant ces mains comme un symbole ou une incarnation de sujet avec qui elle partage un espace publique en prenant le métro.

La page d'accueil de cette œuvre numérique (fig. 9) se compose de photos de mains des passagers du métro sous-titrées et le lecteur peut cliquer sur l'image en miniature pour accéder à l'image en grand format et lire le commentaire de l'auteure qui se trouve au dessous de l'image. En regardant les photos, les auteurs/internautes partent de leur impression pour aboutir aux textes qui permettent d'en revenir encore une fois aux photos. Cette chronologie laisse penser que la contemplation poétique vient au premier rang et le commentaire narratif en découle.

\section{À MAINS NUES}

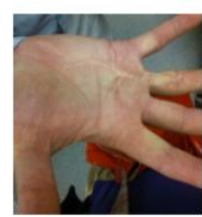

Ouvrir

Opiniâtre

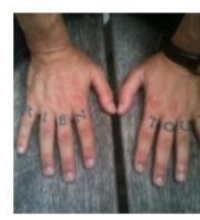

Entre

Ce qui n'est pas visible

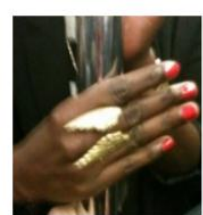

Déplacements

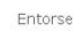

Fig. $(9)^{\mathrm{xv}}$

Parfois le texte est un récit de façon évidente, comme par exemple le texte de l'entrée intitulée «déplacements», montrant une image d'une main de jeune femme avec un grand anneau à double aile qui ressemble, selon Portier, aux ailes d'Hermès, dieu des voyageurs et des échanges (fig. 10). 


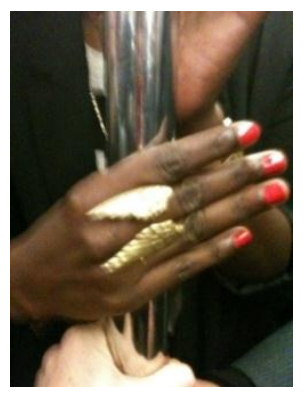

Fig. $(10)^{\mathrm{xvi}}$

«Comme la pie je me suis laissée attirer par le brillant du bijou. Alors j'ai quémandé d'en prendre la photographie, et c'est un étonnement toujours, une belle surprise, de constater la bienveillance avec laquelle mes requêtes déplacées sont accueillies. De bonne grâce elle s'est prêtée à plusieurs poses, mais nous étions dans le remous du métro, et je n'ai réussi à recueillir que cet envol flou. C'était le moins, quand même, que j'arrive à croiser un jour, en plein transport, les ailes d'Hermès, dieu des voyageurs et des échanges. Qu'elles aient volé des semelles du dieu au doigt d'une jeune femme c'est bien normal, puisque les voyageurs de notre temps se déplacent en gardant les pieds immobiles. Reste, quand même, la possibilité de faire circuler la parole, et qu'elle parte là où on ne l'attendait pas [...]»

Dans cet exemple, l'auteure raconte la discussion avec la femme possédant la bague à travers un langage poétique plein de métaphores et d'images. Cette prose poétique caractérise les œuvres de Portier qui emploie les formes courtes poétiques riche de détails et de figure de style.

\section{Conclusion}

Le numérique a modifié l'écriture et la pratique stylistique de C. Portier dans la mesure où le numérique, selon elle, n'est pas seulement un medium ou un outil, mais il constitue un nouveau domaine à investir et à questionner. Il lui permet de créer en ligne 
des formes de poétiques particulières et diverses. Dans La tête que ça nous fait, Portier a exploré nos visages intérieurs qui reflètent nos sentiments les plus intimes. Cette œuvre numérique se caractérise par la finesse de l'expression, la précision de la description, la clarté de vision et le langage parsemé d'images et de métaphores dessinant ainsi une poétique de l'intime mise en place pour représenter la diversité des nos sentiments intérieurs qui se reflètent sur nos visages.

Dans ses œuvres numériques, Dans le viseur, Étant donnée et Traque Traces, Portier a mis en relief la spécificité des traces numériques dans la mesure où nos vies s'inscrivent par trace qui est un genre d'inscription, de présence et d'existence. Dans le viseur tisse une relation immanente entre image et texte : le texte donne vie à l'image et non l'inverse. À travers des textes poétiques fictifs, ce projet numérique réinvente des vies à base des traces laissées. Étant donnée, récit transmédia sous forme de site web, tente de laisser des blancs, de transférer des traces et de poétiser cette fiabilité pour la déjouer. La fiction urbaine Traque Traces emploie le même procédé poétique qui convertit la cartographie numérique en technique littéraire et poétique où Portier a intégré la fonction de Google Maps dans la structure littéraire des personnages et la relation entre eux, afin de produire une œuvre numérique qui regroupe l'espace physique des personnages, l'espace de navigation Web et l'espace de discours narratif.

En observant un univers plutôt prosaïque à travers un regard poétique, Compléments d'objet produit une nouvelle façon de comprendre le monde en donnant une existence et une valeur individuelle aux objets, en invitant les lecteurs à regarder autrement les choses qui les entourent et à les intégrer dans leur quotidienneté non plus en fonction de leurs habitudes, mais en questionnant l'objet et sa fonction. Quant à $\grave{A}$ mains nues, cette 
œuvre rassemble plusieurs photos prises par l'auteure des mains des passagers du métro et raconte ses rencontres inattendues avec eux en leur permettant de parler de leurs mains. Le caractère poétique de ce projet numérique découle de la contemplation de l'auteure et ses rencontres potentielles avec les passagers dans le métro.

Portier exploite ainsi les possibilités numériques pour construire de l'espace à travers une conception innovante de la construction spatiale en donnant une dimension sinon physique, sûrement spatiale à ses œuvres et en employant des éléments littéraires et poétiques tout en rendant iconiques les codes de la cartographie numérique contemporaine. Cette auteure, grâce à la technologie numérique crée des formes poétiques singulières en ligne qui impliquent nos vies, le monde qui nous entoure, notre réalité et nos traces. 
$\underline{\text { Notes : }}$

i Nous désignons par «écrits numériques », les écrits de Portier qu'elle a conçus et publiés exclusivement en ligne.

ii À propos de ce terme «auteur numérisé », numérisé signifie ici avoir une version numérique de ses œuvres en papier.

iii Nous citons à titre d'exemple J.-P. Balpe, Pierre Ménard, Serge Bouchardon, Juliette Mézenc, Philippe Aigrain, Philippe de Jonkheere, Chloé Delaume.

${ }^{\text {iv }}$ Capture-écran, page d'accueil du site d'écriture de C. Portier (petiteracine.net)

$\mathrm{v}$ Notons que cette fleur a la réputation parmi les magiciens et les sorciers d'éveiller l'amour grâce à ses qualités aphrodisiaques et de guérir la stérilité des femmes. Le caractère magique de la mandragore vient principalement de la forme de sa racine qui ressemble vaguement à un corps humain; elle est dotée de deux «jambes » et ses radicelles rappellent des poils. Cela explique pourquoi parmi les nombreux noms qui lui ont été attribués au cours de l'histoire et dans des régions variées de la planète, tous font généralement référence soit à l'amour soit à sa forme humaine. Cette plante incarne parfaitement la rencontre entre l'homme, le végétal et la littérature (le romantisme principalement). Théophile Gauthier, Ludwig Tieck, E.T.A. Hoffmann, Achim von Arnim et Charles Nodier ont trouvé dans cette plante un thème littéraire exceptionnel qui les a conduits à s'interroger sur les liens de l'homme avec la nature. (G. Schaufelberger, G. Vincent, 2004 :140)

${ }^{v i}$ Capture-écran, page d'accueil de La tête que ça nous fait (petiteracine.net)

vii Capture-écran, page d'accueil de Dans le viseur (petiteracine.net).

viii Capture-écran, Dans le viseur (2012-2015), rubrique «Immobile », (petitracine.net).

ix Même si le site consacré au projet de Cécile Portier n'est plus disponible en ligne sur le blog de l'auteure, on peut avoir accès à la version archivée par la Wayback machine d'" Archive.org " à l'adresse suivante $\mathrm{http} / / \mathrm{web}$.archive.org/web/20170915055439/http///etantdonn ee.net/, consulté le 16-4- 2019.

${ }^{\mathrm{x}}$ Capture-écran, page d'accueil d'Etant donnée (petiteracine.net). 


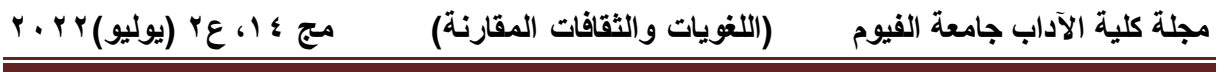

${ }^{x i}$ Le site «Traque traces, une fiction » n'est plus accessible à présent sur le site de l'auteure, cependant il est archivé par Archive.org et disponible en ligne à l'adresse suivante :

https://web.archive.org/web/20160314105711/http://petiteracine.net/tra quetraces/, consulté le 5-7- 2019.

xii Capture-écran, page d'accueil de Traque Traces (2011)

(petiteracine.net)

xiii Capture-écran, page d'accueil de Compléments d'objet (petiteracine.net).

xiv «Les inaperçus », Compléments d'objets, (petiteracine.net).

${ }^{\mathrm{xv}}$ Capture-écran, page d'accueil de À mains nues (petiteracine.net).

${ }^{x v i}$ Capture-écran, rubrique «Déplacements » (2011), À mains nues (petiteracine.net). 


\section{Bibliographie :}

\section{Corpus}

Petite Racine, site Internet de Cécile Portier, www.Petiteracine.net.

\section{Articles écrits par Cécile Portier}

- PORTIER, Cécile. (2-3-2015). «Régime de la trace».

Disponible en ligne :

https://livre.ciclic.fr/actualites/regimes-de-la-trace.

Consulté le 4-1-2021.

- PORTIER, Cécile. (Juillet 2016). «Étant donnée : une fable poétique sur le régime de notre identité numérique », Itinéraires [En ligne]. 2015-3| 2016.

Disponible en ligne :

http://journals.openedition.org/itineraires/3124. Consulté le 9-5-2021.

- PORTIER, Cécile. (s. d. "A"). «Cécile Portier, (auto)portrait ». Disponible en ligne : http://www.ciclic.fr/livre-lecture/residencesnumeriques/cecile-portier-autoportrait. Consulté le 2-32021.

- PORTIER, Cécile. (s. d. "B"). «Quel fil pour recoudre des passés disloqués? ». Disponible en ligne :

http://livre.ciclic.fr/actualites/quel-fil-pour-recoudre-despasses-disloques. Consulté le 4-1-2021.

- PORTIER, Cécile. (s. d. "C"). « Vestiges de soi, lignes d'insu ». Disponible en ligne : http://livre.ciclic.fr/vestigesde-soi-lignes-d-insu-une-residence-pour-interroger-nostraces-d-images-et-de-donnees. Consulté le 5-4-2021. 


\section{Articles consacrés à Cécile Portier}

- BOUTOUILLET, Guénaël. (17 mars 2011). «Traque Traces. Un site de Cécile Portier ». Disponible en ligne : https://remue.net/traque-traces-un-site-de-cecile-portier4175. Consulté le 6-7-2021.

- BOUTOUILlET, Guénaël. (4-11-2015). «Cécile Portier, une auteure en présence sur ciclic.fr ». Disponible en ligne : http://livre.ciclic.fr/actualites/cecile-portier-uneauteure-en-presence-sur-ciclicfr. Consulté le 9-3-2021.

- LEFEBVRE-CÔTÉ, Béatrice. (2018). «Produire l'espace du quotidien : Compléments d'objets de Cécile Portier ». Sens public. Disponible en ligne :

https://doi.org/10.7202/1059040ar. Consulté le 28-4-2021.

- DEBEAUX, Gaëlle. (2019). «Lignes de fuites poétiques du récit numérique ». Usages, formes et enjeux de la « poésie numérique». Revue en ligne du Rirra 21. Textes réunis par Claire Chatelet et Gwendolyn Kergourlay. Vol. $\mathrm{n}^{\circ}$ 12. Disponible en ligne : http://komodo21.fr/lignes-defuites-poetiques-recit-numerique/. Consulté le 9-5-2019.

- MARCHESE, Enrico Agostini. (Octobre 2020). « Ecrire l'espace : dynamiques d'imbrication performative de la photographie dans la littérature numérique ». Cahiers de Marge, $\mathrm{n}^{\circ} 2$. Disponible en ligne :

https://papyrus.bib.umontreal.ca/xmlui/bitstream/handle/18 66/24045/. Consulté le 17-5-2021.

\section{Articles généraux :}

- DESLAURIERS, R. (2002). Les Meidosems d'Henri Michaux : émergences du dedans, résurgences orientales. Tangence, (68), 121-136. Disponible en ligne : https://doi.org/10.7202/008252ar. Consulté le 3-1-2021. 


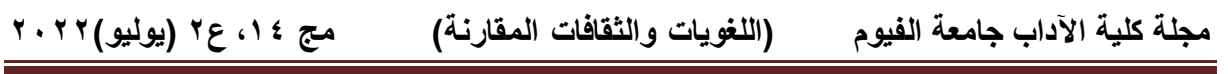

- SCHAUFELBERGER, Gilles. VINCENT, Guy. (2004). Le mythe de la Mandragore, la «plante-homme » (dossier et extraits). Diogène. 2004/3, n 207, pages 140 à 173.

Disponible en ligne : https://www.c airn.info/revuediogene-2004-3-page-140.htm. Consulté le 8-3-2021.

- SIAUGUES, Fanny. (2019). «Un aménagement réflexif des formes textuelles », Elfe XX-XXI, Revue en ligne. $8 \mid$ 2019. Disponible en ligne :

https://doi.org/10.4000/elfe.1225. Consulté le 5-2-2021.

V. Entretint avec Portier

- Entretien avec Cécile Portier, à propos de son ouvrage De toutes pièces. 05/10/2018. Disponible en ligne : https://www.babelio.com/auteur/Cecile-Portier/46754. Consulté le 3-4-2021. 